\title{
Decoupling of ground level pressures observed in Italian volcanoes: are they driven by space weather geo-effectiveness?
}

\author{
Paolo Madonia ${ }^{1, \star}$, Paolo Romano ${ }^{2}$, Salvatore Inguaggiato $^{1}$ \\ ${ }^{1}$ Istituto Nazionale di Geofisica e Vulcanologia, Sezione di Palermo, Italy \\ ${ }^{2}$ INAF- Osservatorio Astrofisico di Catania, Italy
}

\author{
Article history \\ Received June 29, 2012; accepted June 18, 2014. \\ Subject classification: \\ Atmospheric pressure, Space weather, Sun storm, Solar-terrestrial interaction, Volcanoes, Volcanic effects.
}

\begin{abstract}
Investigations on correlation drops between near-ground atmospheric pressures measured at sea level and at higher altitudes on Italian volcanoes have been carried out. We looked for perturbations of the atmospheric pressure field driven by volcanic activity, but not excluding possible external triggers for the observed anomalies. Decorrelations between atmospheric pressures measured at Stromboli Island in stations located at different altitudes (years 2002-10) have been analysed and compared with data from other volcanic (Vesuvius) and non volcanic (Mt. Soro) orographic structures. We investigated as their possible triggers volcanic, meteorological and space weather parameters, with particular attention to Total Solar Irradiance (TSI), Kp index and Forbush decreases. Pressure decorrelations seems to be driven by astronomic cycles, with maxima in summer and minima in winter. A further contribution was found, seemingly assignable to TSI anomalies, with correlation minima occurring 12 hours after these but only during phases of high Sun activity. Moreover, during the same phases a main periodicity of about 27 days in pressure decorrelations was revealed by FFT analysis. This period is the same of the Sun Carrington rotation, expressing the periodic reappearance of sunspot groups on Sun's surface. The strong similarity between recurrences of sunspot number and atmospheric pressure anomalies further supports the role of the former as a possible trigger for the latter.
\end{abstract}

\section{Introduction}

The influence of space weather on Earth systems is not easy to be investigated: the main difficulty in the recognition of proxies for space weather geo-effectiveness is the need of a close interplay between distinct scientific communities, with different educational backgrounds and languages, very rarely working in multidisciplinary, equally-ranked teams. The discovery of new evidence of Sun-Earth connections is then sometimes entrusted to the accidental evolution of researches, aimed $a b$ initio to different goals, but pointing at their end to the conclusion that an Earth system shows an external forcing seemingly assignable to the geo-effectiveness of space weather. The analysis and interpretation of ground level atmospheric pressure anomalies recorded in volcanic surveillance networks is paradigmatic of such a concept.

Hourly data of atmospheric pressure are normally recorded at ground level on Italian volcanoes (Vesuvius and Stromboli; see Figure 1) as a side parameter for environmental and volcanic hazard monitoring purposes. Due to the particular geometry of volcanic edifices, conically shaped with steep flanks, the data acquisition networks are characterized by the presence of couples of measuring sites with huge altitude differences but closely spaced in terms of planar distances.

During the 2002-03 Stromboli eruption, Brusca et al. [2004] identified wind and air temperature anomalies on the top of the volcano driven by volcanic activity. A retrospective analysis of these data has been carried out looking for other independent proxies of the observed anomalies; in particular, attention has been focused on atmospheric pressure, supposing that this parameter could had been influenced by circulation of hot air due to volcanic activity. The initial hypothesis was that, since atmospheric pressure is temperature dependent, the variable thermal activity from the summit vents should induce atmospheric pressure transients limited to their immediate (hundreds of meters) surroundings. No volcanic effects are expected at sea level, because here we are out of the influence of the summit vent thermal field. If this hypothesis was true, i.e. if instant pressure values at vent level (influenced by volcanic activity) were different with respect those recorded at sea level, a poor correlation between them should be observed. In order to test this hypothesis we compared atmospheric pressure measures at Stromboli 


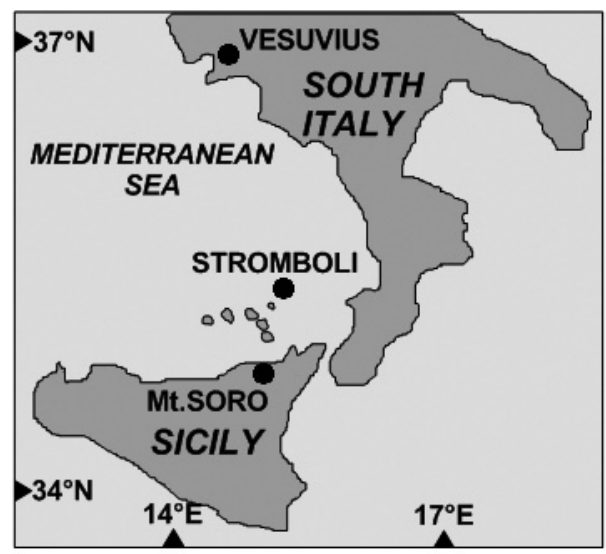

Figure 1. Location map of the studied areas.

with other data recorded both in volcanic (Vesuvius) and non volcanic (Mt. Soro, Sicily) areas, extending the observational period for several years. We initially looked for meteorological proxies of volcanic activity but, following the first outcomes, we extended our search to possible space weather signatures affecting the atmospheric pressure signal. Due to this huge change of perspective we will present and discuss our data reconstructing, step by step, the logical path we followed in the interpretation of the observed anomalies.

\section{Data collection}

Our dataset covers the period from January 21, 2003 , to September 1st, 2010. It is populated by hourly data of atmospheric pressure, recorded in 6 meteorological stations from 3 different area networks (Figure 1). Data from volcanoes refer to 2 stations at Stromboli (ST) and other 2 at Vesuvius (VE), presently in a quiescent state with no significant degassing and thermal activity; data from Vesuvius are limited to the period June 2003-January 2005. Atmospheric pressures from non volcanic mountains refer to 2 stations of the Sicilian Agro-meteorological Service (SIAS, http: / / www.sias. regione.sicilia.it/) operating at Mt. Soro (SO), a $1814 \mathrm{~m}$ high mountain located close to the east sector of the northern mountain chain of Sicily. Coordinates and

\begin{tabular}{cccc}
\hline Id (area) & Easting & Northing & Elevation \\
\hline STR01 (ST) & 520631 & 4293832 & 10 \\
STR02 (ST) & 518670 & 4294319 & 890 \\
PAT (SO) & 501713 & 4221477 & 70 \\
SOR (SO) & 473142 & 4198383 & 1810 \\
TGR (VE) & 447210 & 4515358 & 60 \\
CRA (VE) & 451559 & 4519368 & 1250 \\
\hline
\end{tabular}

Table 1. Coordinates (UTM WGS84-33S Zone) and elevations (m a.s.l) of atmospheric pressure measuring stations (letter between brackets after Id indicates the area, Mt. Soro, Vesuvius and Stromboli). altitudes of all the stations are summarized in Table 1. The altitude differences of the 3 station twins are: 880 $\mathrm{m}$ for Stromboli, $1190 \mathrm{~m}$ for Vesuvius and $1740 \mathrm{~m}$ for Mt. Soro. Data resolution is $0.1 \mathrm{hPa}$ for Stromboli and Mt. Soro and $1 \mathrm{hPa}$ for Vesuvius.

In order to investigate a possible time correlation between the atmospheric pressure data and some geoeffective Sun parameters we also considered:

- Daily Kp index, which quantifies disturbances in the horizontal component of Earth's magnetic field due to the Sun activity and calculated from a network of geomagnetic observatories. The valuse of the daily $\mathrm{Kp}$ index is published by NOAA at ftp: / /ftp.ngdc.noaa.gov/ STP/GEOMAGNETIC_DATA/INDICES/KP_AP/

- Total Solar Irradiance (TSI) data published by the Physikalisch-Meteorologisches Observatorium Davos (PMOD) at ftp: / /ftp.pmodwrc.ch/pub/ data/irradiance/ composite/DataPlots/composite_d41_62_1110.dat

- Forbush decreases, i.e. the decreases in the observed galactic cosmic ray intensity which follow the solar corona plasma ejections. This parameter is reported by the Pushkov Institute of Terrestrial Magnetism, Ionosphere and Radio Wave Propagation of the Russian Academy of Sciences (IZMIRAN) at http: / / helios.izmiran. rssi.ru/cosray/ events.htm.

We analysed all these data both in time and frequency domains. In the time domain we evidenced pressure anomalies calculating linear regression coefficients between the possible couples of stations from the same network in moving time windows of 6 days. We choose this particular size for the time window because 6 days is the complete duration of tropospheric pressure disturbances driven by Sun storms (the so called "Wilcox effect"; see next chapter for all the details). Data were also analysed in the frequency domain with the FFT algorithm implemented in the freeware KyPlot, version 2.0 (http://www.kyenslab.com/en/).

\section{Data analysis and discussion}

3.1. Volcanic activity, atmospheric air circulation and pressure correlation drops

As a first attempt to evaluate the possible effect of volcanic activity on atmospheric pressures we considered time variations of pressure differentials between top and bottom stations in the three areas (Figure 2). As shown in the figure, they were modulated by seasonal cycles, with maxima in winter and minima during summer, with the superimposition of a high frequency component induced by the short-term meteorological variability. An anomalous feature was observed at Stromboli, characterized by a strong variability of the differentials in the period between September 2003 and February 2004 


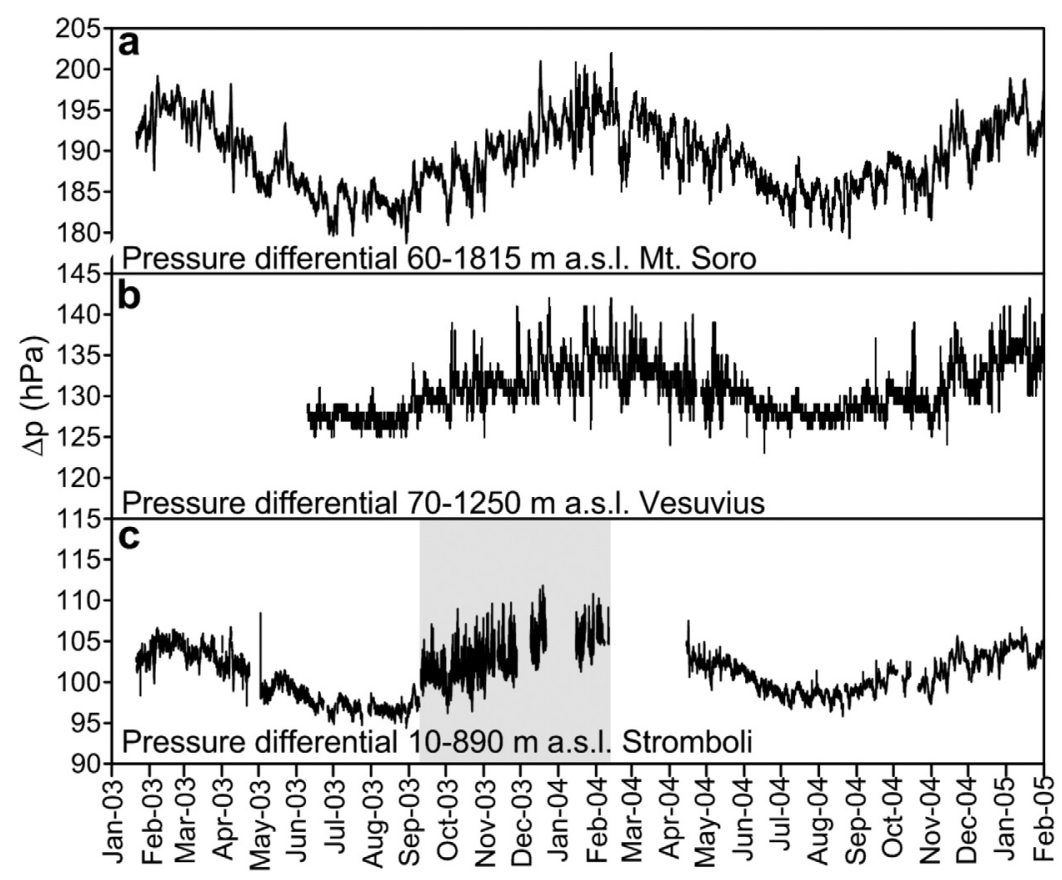

Figure 2. Pressure differentials between hourly values acquired at the bottom and top stations at Mt. Soro (a), Vesuvius (b) and Stromboli (c). The grey band in Stromboli plot highlights the high air turbulence period corresponding to the renewal of the normal activity after the 2002-03 eruption.

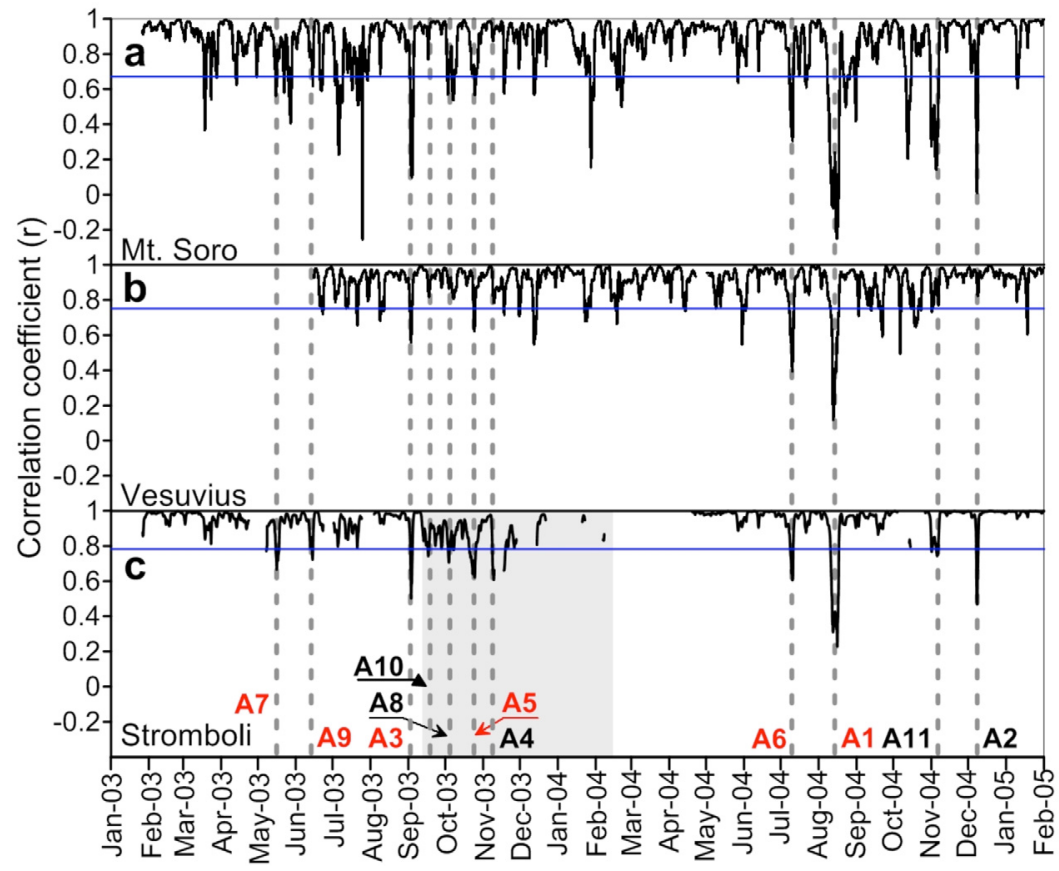

Figure 3. Linear regression coefficients ( $r$ ) between hourly atmospheric pressure values recorded at bottom and top stations at Mt. Soro (a), Vesuvius (b) and Stromboli (c) in the period January 2003-January 2005; moving time window for calculating correlation is set to 6 days (144 measures). The grey band in Stromboli plot highlights the high air turbulence period corresponding to the renewal of the normal activity after the 2002-03 eruption. The grey dashed lines evidence the 11 main drops of correlation (down the thresholds represented by the blue lines and described in the following Figure 4) measured at Stromboli and progressively numbered for decreasing amplitudes; red numbers refer to anomalies simultaneously observed in the three networks.

(grey band in Figure 2c); this period coincided with the renewal of volcanic activity after the 2002-03 Stromboli eruption, suggesting a volcanic-driven effect on the local, near ground atmospheric pressure field.

For supporting this first inference, the Pearson correlation coefficients ( $r$ ) between the two Stromboli sta- tions were calculated and compared with the values related to the other two networks (Figure 3). The general behaviour of the correlation signal in all the considered areas is characterized by several marked correlation drops, emerging from a background scattering whose amplitude is variable among different areas. 


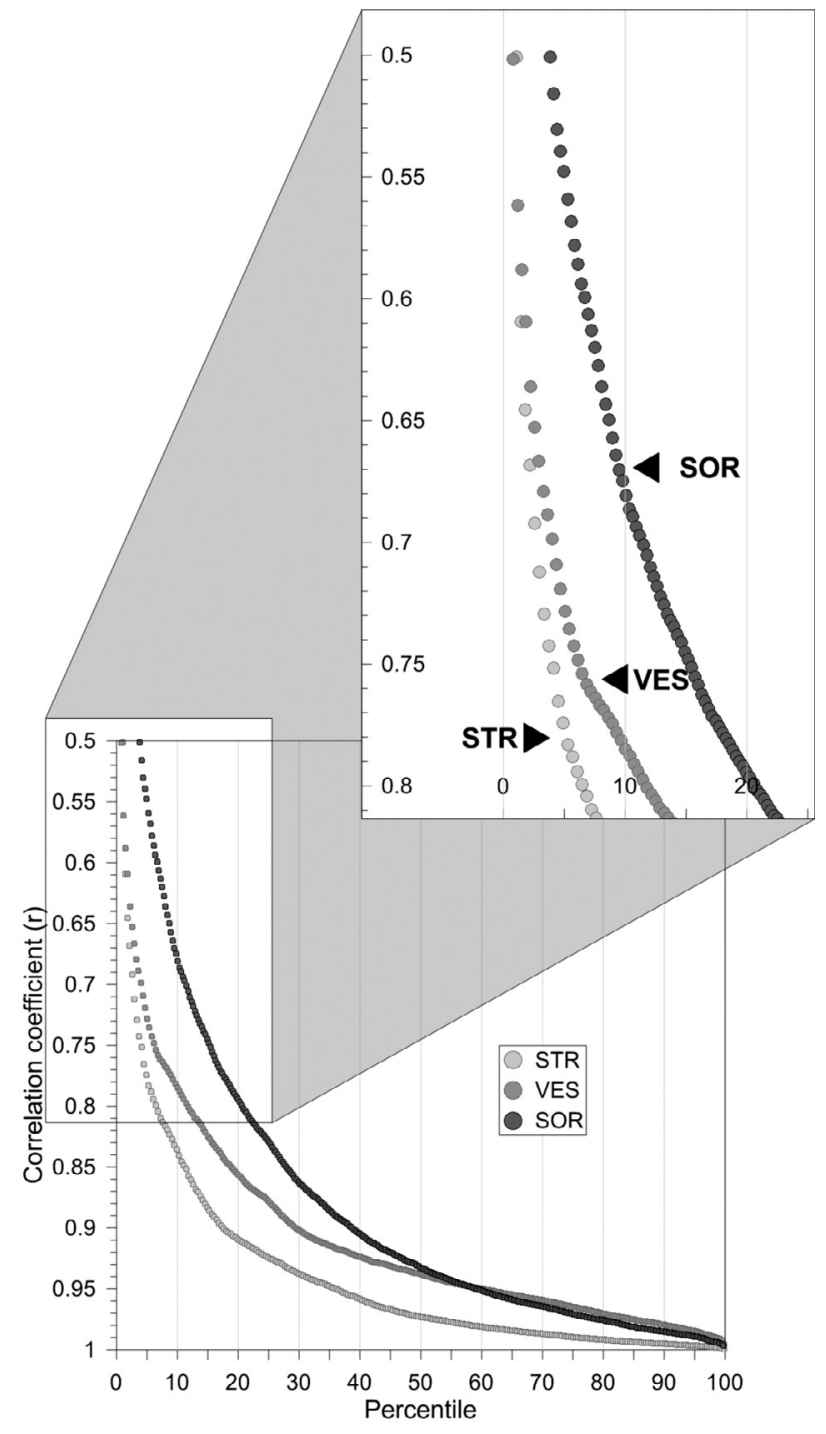

Figure 4. Probability plot of Pearson correlation coefficients between atmospheric pressures acquired at different heights at Stromboli (STR), Vesuvius (VES) and Mt. Soro (SOR). The thresholds used for the individuation of the "main" pressure correlation drops in the different areas are individuated in the exploded inset illustrated in the top right corner of the figure.

Correlation drops (Figure 4) are not linked to random pressure outliers recorded in one or both stations, but to trends diverging on a multi-days time scale between the two measuring sites at different altitudes. This is illustrated in the example of Figure 5, where atmospheric pressure variations recorded at Mt. Soro between August 10 and August 16, 2004, are reported.

We evidence a progressive increase of the background scattering of the correlation signal from Stromboli to Vesuvius and to Mt. Soro. Two concurrent mechanisms may be claimed to explain the increasing correlation scattering, both related to the role of local conditions in disturbing the pressure signal: i) the increasing altitude differences between the station twins $(880,1190,1740 \mathrm{~m}$, respectively) and ii) the progressive complication of the orographic structures. In fact, Stromboli is a thermal island (a simple, conic-shaped relief) surrounded by a homogeneous media (the Sea). Vesuvius is inserted inside one of the most heavily urbanized areas of the world. Mt. Soro is the main elevation of a tens of kilometres-long mountain chain located several kilometres apart the coastline. In other words, the more is complicated the local orography and/or the higher is the difference of altitude (e.g. barometric height) between station twins, the more turbulent the air circulation regime will be, giving raise to pressure correlation drops between different barometric heights.

In order to isolate "main" anomalies with respect to the background noise we used the probability plot of the correlation coefficients for the three areas, illustrated in Figure 4. As shown in the graph, the thresholds for the main anomalies are $0.78,0.75$ and 0.67 for Stromboli, Vesuvius and Mt. Soro, respectively.

Eleven main anomalies $(0.78>$ r, progressively numbered for decreasing amplitude) were individuated at Stromboli (Figure 3c). The main concentration of correlation drops (anomalies A3, 4, 5, 8) was registered during the previous mentioned turbulent period, linked to the renewal of volcanic activity and lasted from September 2003 to February 2004. This observation seems to confirm that volcanic activity can produce atmospheric pressure disturbances at ground level but, since correlation drops occur also outside active volcanic areas, other coexisting mechanisms have to be invoked for explaining the observed anomalies. In particular, a very peculiar feature is the synchronicity of 6 main anomalies (A1, 3, 5, 6, 7, 9), observed in all the areas (we included anomalies A7, 9 occurred in a period not covered by Vesuvius data). Moreover two of these, A3 and A5, occurred during the previously discussed turbulent period at Stromboli. We were then driven to exclude the bi-univocal correspondence between pressure decoupling and volcanic activity and to consider the coexistence of a possible external, wider scale trigger for such anomalies.

The first possible common source for the observed simultaneous correlation drops could be identified in some peculiar atmospheric pressure field acting on the South Mediterranean area. However, as suggested by the analysis of the database of the Italian Air Force Meteorological Service (Teodoro La Rocca, personal communication), during all the observed anomalies a normal meteorological regime dominated on the South Mediterranean, without dominant pressure configurations different with respect to adjacent time windows. As a consequence, we excluded the effect of regional meteorological phenomena.

With the aim of looking for other relationships between possible peculiar atmospheric pressure configurations and de-correlations, Pearson correlation coefficients were then plotted, year by year, against pressure 


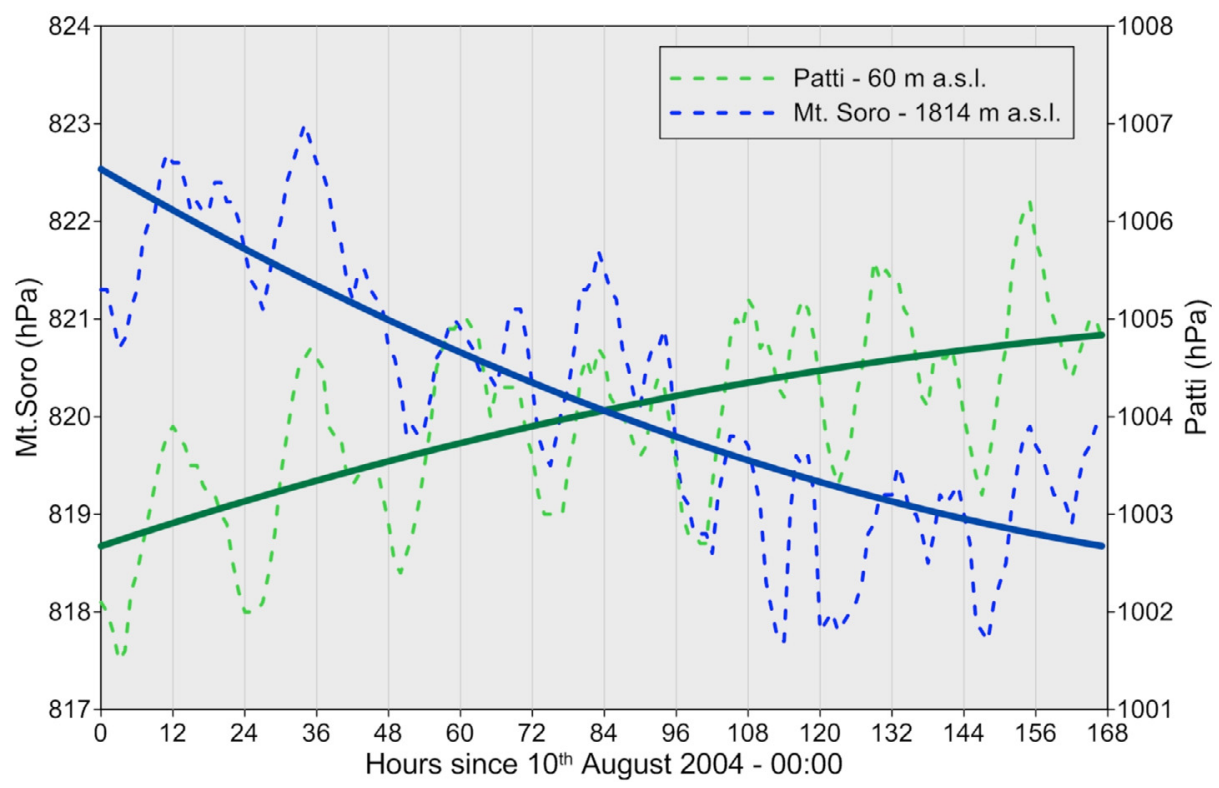

Figure 5. Atmospheric pressure recorded at Mt. Soro between August 10 and August 16, 2004. Dashed lines refer to hourly data from Patti (green) and Mt. Soro (blue), thick continuous lines are their 2nd degree polynomial best fits.

values recorded in each station. Data distributions were quite similar in all the locations: we reported in Figure 6 as a common example the results from Stromboli STR01 (sea level) station. Even if with some differences both in term of absolute values and relative distributions, pressure correlation coefficients showed prominent minima centred around a typical pressure value, that at STR01 varied in a narrow range comprised between 1010 and $1015 \mathrm{hPa}$. Yearly data from all the stations are reported in Figure 7, where pressure data at which minima of pressure correlations occurred are plotted against altitudes of the measuring locations. Vertical pressure gradient obeys to the exponential barometric formula:

$$
P_{h}=P_{0} e^{-m g h / k T}
$$

where $P_{h}$ and $P_{0}$ are the pressures at sea level and at a certain altitude $h$ respectively, $m$ the total mass of the considered air volume, $g$ the gravity acceleration, $k$ the Boltzmann's constant and $T$ the absolute temperature of air. Since the altitude differences between the stations is low, a simplified linear fit well approximates the theoretical curve. Its good correlation coefficient demonstrates that vertical variations of pressures at which minima of correlations occurred in the different stations are only driven by the ratio $-h / T$. The intercept at sea level (1013-1014 $\mathrm{hPa})$ is very close to the value of $1010 \mathrm{hPa}$, considered as the divide between high and low atmospheric pressures. Therefore, we noted that minima of correlation are more frequent during condition of "neutral" pressures, i.e. when the absence of oriented atmospheric pressure fields lets air circulation free to be driven by other mechanisms.
Among the other possible triggers for the observed anomalies we also considered space weather, as detailed in the next section.

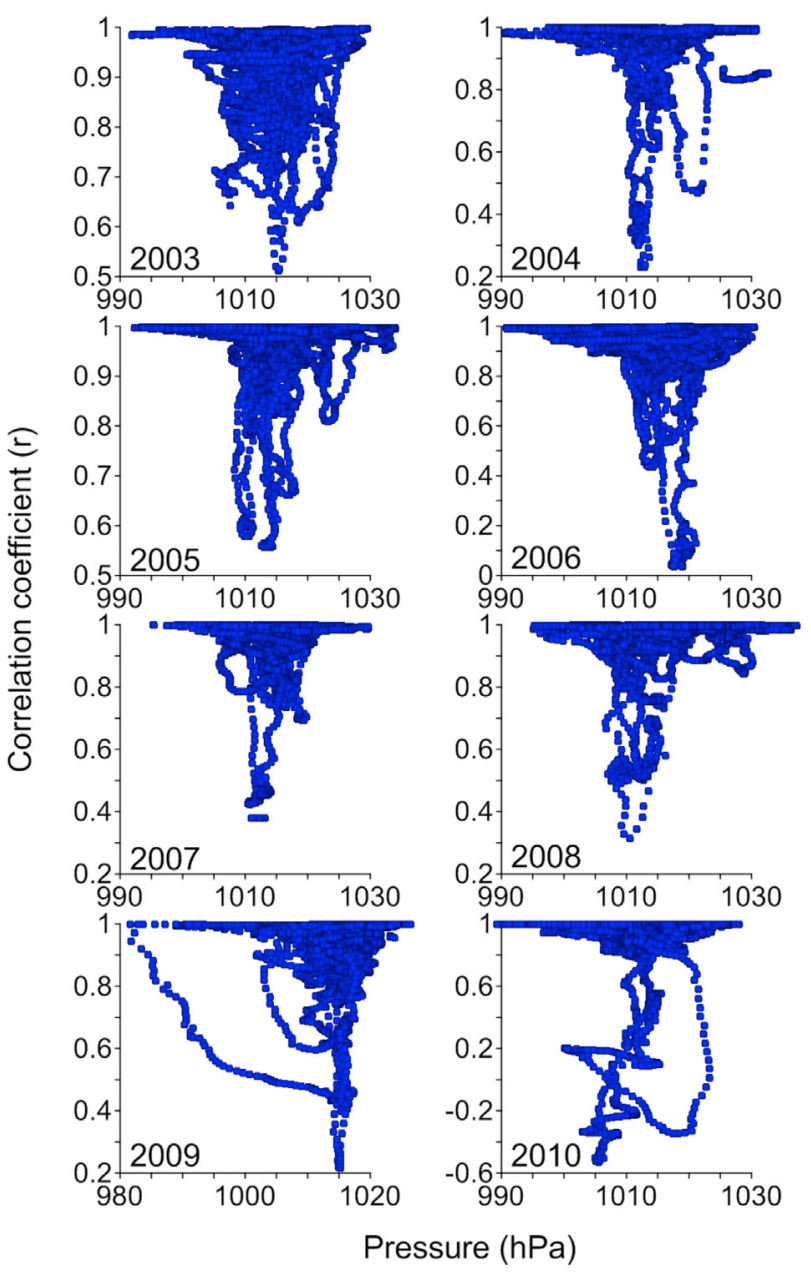

Figure 6. Pressure correlation coefficients calculated at Stromboli plotted versus hourly values of atmospheric pressure recorded at STR01 station (years 2003-10). 


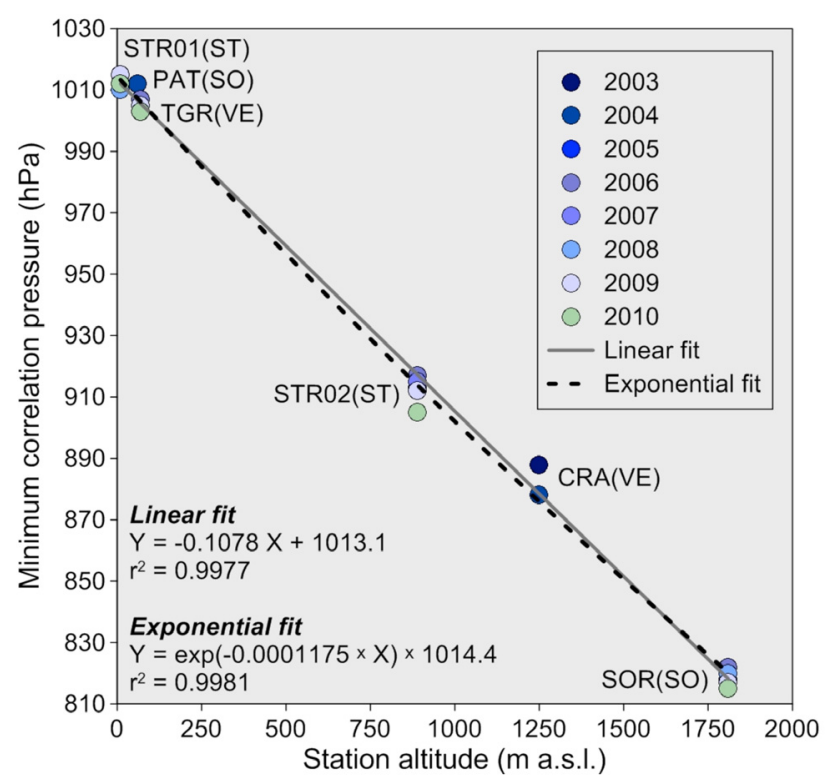

Figure 7. Yearly values of pressures at which minimum correlations between stations occurred plotted versus altitude of the stations.

\subsection{Space weather and pressure correlation drops}

The possible influence of space weather on the Earth atmospheric pressure field was proposed by Macdonald and Roberts [1960] and further supported by Wilcox et al. [1974]: the formation of wintertime lowpressure troughs, developing or moving into North $\mathrm{Pa}-$ cific region and showing anomalous deepenings 2-4 days after a bright aurora or a geomagnetically disturbed day, were related to the passing over the Earth of the solar magnetic sector structures of the Interplanetary Magnetic Field (IMF). Since the publication of the cited study these anomalies have been referred as the "Wilcox" effect. Later researches gave controversial explanation about the physical mechanisms responsible for the Wilcox effect. Some authors attributed pressure anomalies to the injection of volcanic aerosols into the stratosphere and the consequent induced changes in current density of the global electric circuit and in relativistic electron precipitation, on their turn driving perturbations in atmospheric dynamics due to ice nucleation, precipitation efficiency and latent heat retention [Tinsley et al. 1994, Kirkland et al. 1996, Mironova et al. 2011, Tinsley et al. 2012]. On the contrary, Prikryl et al. [2009a,b] revisited the original Wilcox's data, suggesting that pressure anomalies concern the northern as well as the southern hemisphere winters during periods both of low and high volcanic aerosol loadings. They invoked solar wind generated auroral atmospheric gravity waves (AGWs) as modulators of cyclogenesis and responsible for significant sea level pressure deepenings of extratropical storms.

Looking at Sun-Earth interactions as a possible cosource for the pressure anomalies we observed, we focused our attention to the indexes of those phenomena

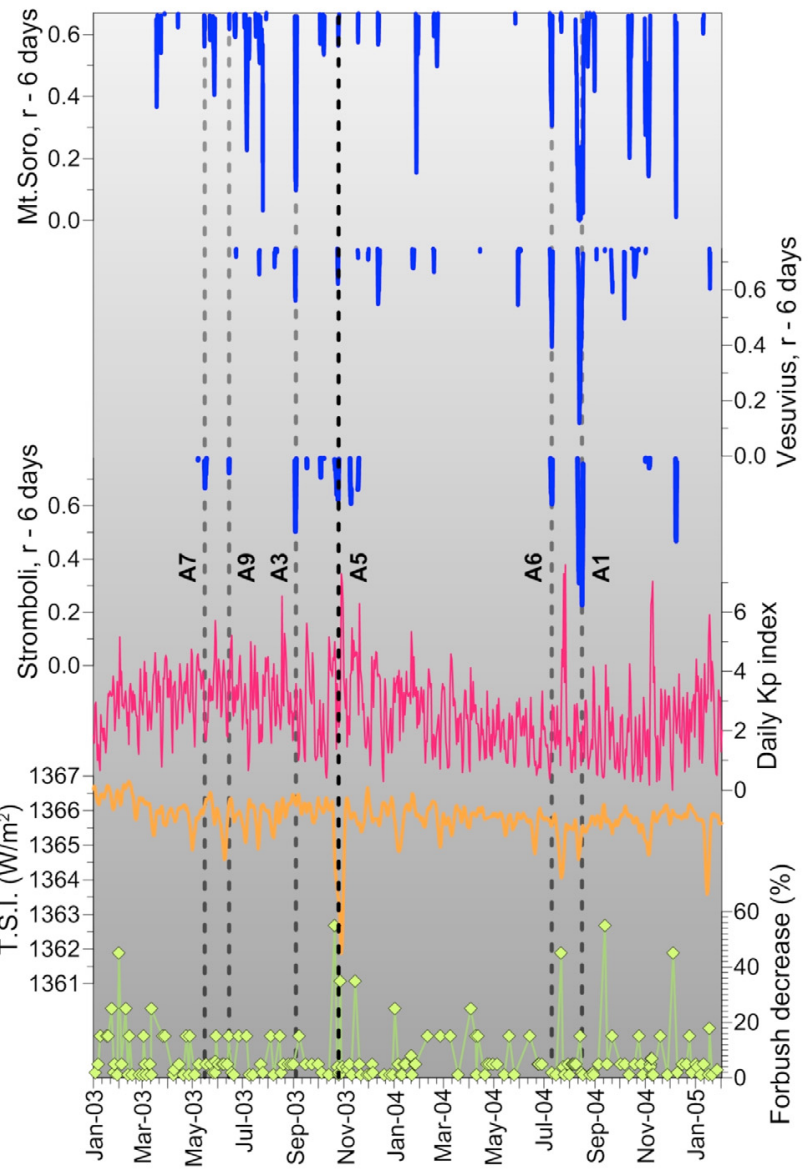

Figure 8. From the top to the bottom: pressure linear correlation coefficients ( $\mathrm{r}$ calculated on 6 day moving time windows, only values over the thresholds described in Figure 4) measured at Mt. Soro, Vesuvius and Stromboli (base cut-off set at 0.8 for removing background noise) in the period January 2003-January 2005, daily values of Kp index, daily values of Total Solar Irradiance (T.S.I.) and Forbush decreases. The grey dashed lines evidence the six main drops of correlation contemporaneously measured in all the networks (Figure 3).

potentially able to influence atmospheric turbulence. Based on the previously cited literature models, we considered the parameters linked to the Earth's Global Electric Circuit and Magnetic Field: the Total Solar Irradiance (TSI), the Kp indexes of the geomagnetic field activity and the Forbush decreases (FD).

We first considered the above mentioned data in the time domain, plotting these together with pressure correlation coefficient drops in Figure 8. We used the same cut-off values of Figure 3 for evidencing the main anomalies, and concentrated our attention only on those six simultaneously occurred in the three study areas, indicated in the figure by the vertical dashed lines.

At a first, eyeballing look pressure anomalies seem to be observed in time relationship with changes in Sun activity, even if neither a direct cause-effect link or a proportionality between Sun activity and pressure anomalies can be stated out of any doubt. Since our observations began two years after the maximum of the XXIII solar cycle (2001), we were driven to verify if sim- 


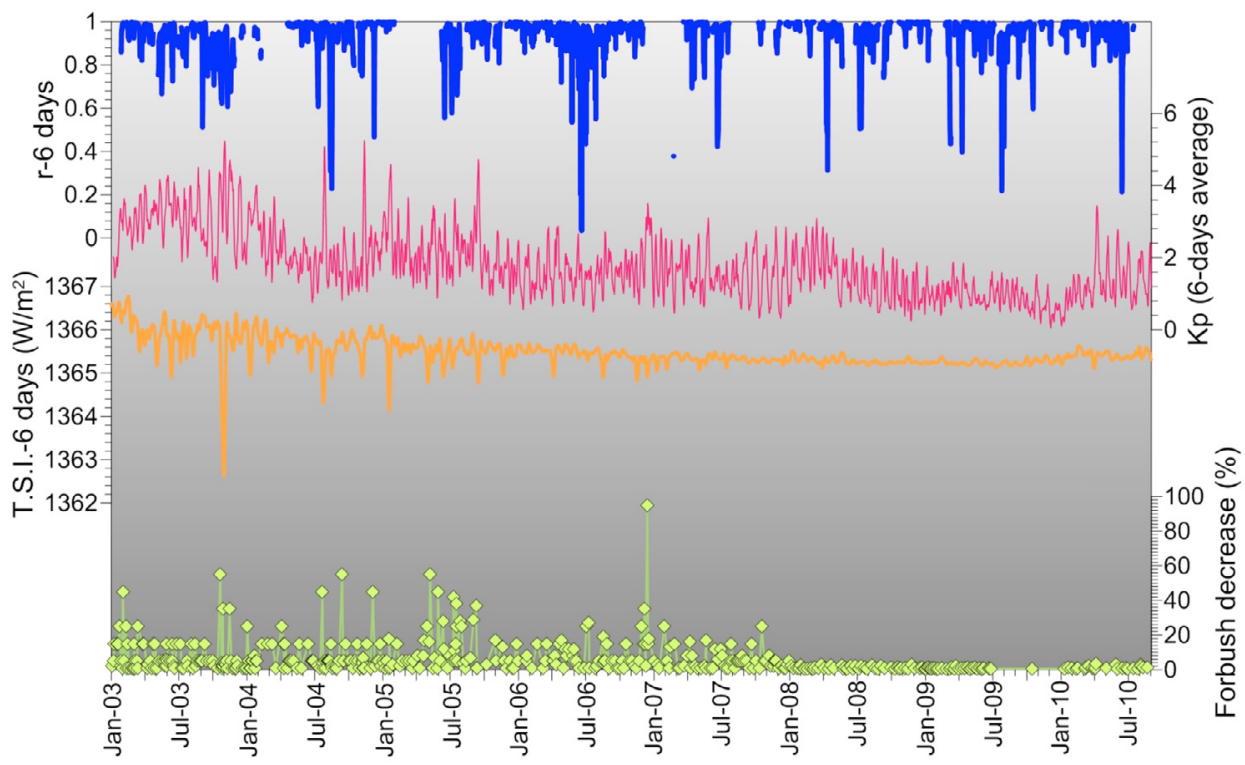

Figure 9. From the top to the bottom: pressure linear correlation coefficients ( $\mathrm{r}$ calculated on 6 days moving time windows) measured at Stromboli in the period January 2003-June 2010, 6-day averages of Kp index, 6-day averages of Total Solar Irradiance (T.S.I.), Forbush decreases.

ilar anomalies were observed also under quieter Sun conditions. In the graph of Figure 9 we reported pressure correlation coefficients, calculated at Stromboli, compared to 6-day averaged Kp indexes, TSI and FDs for the period January 2003-June 2010. The highest significant drops in correlation seem to occur with a seasonal cyclicity around the summer solstice for the northern hemisphere, when the solar irradiance is at its relative maximum. Moreover, correlation drops seem to occur both under quiet and very active Sun conditions, thus excluding a direct influence of the quasi-11 yr Sun cycles. However, a more accurate analysis of the figure reveals some deviations from the above discussed general pattern. In the year 2003 correlation minima were shifted toward autumn, in correspondence of $\mathrm{Kp}$, TSI and FD anomalies. In 2004 a secondary correlation minimum was observed on November, again during enhanced Sun variability. In 2008 another correlation minimum was observed on March, during high Kp. In the last two years (2009-10), characterized by very quiet Sun conditions, no anomalous correlation drops (out of the seasonal cycle) were detected.

For testing this hypothesis we moved our focus from pressure decorrelation to Sun variability. Fixed at 0 the day of the TSI and FD anomalies, we considered a time window from 6 days before to 6 days after the day of the anomaly (13 days in total), computing the variation of pressure correlation coefficients with the same criteria discussed in the method chapter. The complete lists of the main TSI anomalies and FDs with amplitudes higher than $15 \%$ and the related minima and maxima of pressure correlation coefficients recorded in the three networks are reported in Tables 2 and 3 respectively. With the aim of removing random noise and enhancing possible common time patterns of pressure correlation coefficients, we carried out a superposed epoch analysis of the 37 TSI and 27 FD anomalies, whose results are illustrated in Figures 10 and 11 respectively. Data were separated in two distinct periods, from January 2003 to July 2005 the first one and from August 2005 to June 2010 the second one, in order to highlight the possible effect of different Sun activity states.

The superposed epoch analysis of the TSI anomalies (Figure 10) clearly identified very different time patterns of pressure correlations in the two periods. During the first period, just after the maximum of the solar cycle, in all the three areas pressure correlations followed the same pattern of TSI anomalies. In particular, both the pressure correlations and the TSI anomalies decrease in 6 days toward a common minimum reached by the former with a time delay of about 12 hours with respect to the latter. Both parameters resumed their pristine values during the 6 days after their minima. On the opposite during the following period, under a much more quiet Sun, pressure correlations followed a random pattern completely independent with respect to TSI, with fluctuations not comparable between the two areas (Stromboli and Mt. Soro) for which data were available.

Not so unambiguous is the interpretation of the correlation signal linked to FDs (Figure 11). During the first period the common feature observed in all the areas was a very pronounced minimum of correlation occurred 72 hours after the main FD, that was anticipated 7 days before by a secondary anomaly with an amplitude of about $20 \%$ of the main decrease. Other decorrelations occurred during the 7 days elapsed between the secondary and the main FDs, with minor 
MADONIA ET AL.

\begin{tabular}{|c|c|c|c|c|c|c|c|}
\hline Date & $\begin{array}{l}\text { TSI minimum } \\
\left(\mathbf{W} / \mathbf{m}^{2}\right)\end{array}$ & $\begin{array}{c}\mathrm{r}-\mathrm{min} \\
(\mathrm{SO})\end{array}$ & $\begin{array}{c}\mathrm{r}-\mathrm{max} \\
(\mathrm{SO})\end{array}$ & $\begin{array}{c}\mathrm{r}-\mathrm{min} \\
(\mathrm{ST})\end{array}$ & $\begin{array}{c}\mathrm{r}-\mathrm{max} \\
(\mathrm{ST})\end{array}$ & $\begin{array}{l}\text { r-min } \\
(\mathrm{VE})\end{array}$ & $\begin{array}{c}\text { r-max } \\
(\mathrm{VE})\end{array}$ \\
\hline 22_Feb_03 & 1365.74 & 0.88 & 1.00 & 0.91 & 1.00 & n.d & n.d \\
\hline 15_Mar_03 & 1365.28 & 0.37 & 0.95 & 0.84 & 0.99 & n.d & n.d \\
\hline 01_May_03 & 1364.86 & 0.67 & 0.98 & n.d. & n.d & n.d & n.d \\
\hline 09_Jun_03 & 1364.58 & 0.62 & 0.99 & 0.73 & 0.99 & n.d & n.d \\
\hline 05_Jul_03 & 1365.08 & 0.23 & 0.98 & n.d & n.d & 0.77 & 0.99 \\
\hline 20_Jul_03 & 1364.89 & -0.26 & 0.96 & 0.79 & 0.97 & 0.66 & 0.97 \\
\hline 08_Aug_03 & 1365.21 & 0.69 & 0.99 & 0.93 & 1.00 & 0.68 & 0.97 \\
\hline 29_Oct_03 & 1361.87 & 0.56 & 0.98 & 0.62 & 0.97 & 0.62 & 0.97 \\
\hline 22_Nov_03 & 1365.39 & 0.58 & 0.99 & 0.68 & 0.92 & 0.71 & 0.98 \\
\hline 07_Jan_04 & 1364.82 & 0.87 & 1.00 & n.d & n.d & 0.91 & 0.99 \\
\hline 26_Feb_04 & 1364.94 & 0.50 & 0.99 & n.d & n.d & 0.81 & 0.98 \\
\hline 11_Mar_04 & 1365.28 & 0.77 & 0.97 & n.d & n.d & 0.80 & 0.99 \\
\hline 15_May_04 & 1365.28 & 0.80 & 0.99 & 0.98 & 1.00 & 0.75 & 0.96 \\
\hline 24_May_04 & 1365.45 & 0.64 & 0.99 & 0.89 & 1.00 & 0.55 & 0.98 \\
\hline 20_Jun_04 & 1364.75 & 0.91 & 0.99 & 0.97 & 1.00 & 0.90 & 0.98 \\
\hline 22_Jul_04 & 1364.05 & 0.61 & 0.99 & 0.88 & 1.00 & 0.83 & 0.99 \\
\hline 12_Aug_04 & 1364.59 & -0.25 & 0.93 & 0.23 & 0.99 & 0.12 & 0.97 \\
\hline 22_Sep_04 & 1365.33 & 0.75 & 0.99 & 0.87 & 0.99 & 0.59 & 0.99 \\
\hline 05_Nov_04 & 1364.72 & 0.14 & 0.98 & 0.75 & 1.00 & 0.73 & 0.98 \\
\hline 15_Jan_05 & 1363.58 & 0.60 & 0.99 & 0.95 & 1.00 & 0.60 & 0.99 \\
\hline 30_Apr_05 & 1364.53 & 0.16 & 0.97 & n.d & n.d & n.d & n.d \\
\hline 10_Jun_05 & 1364.76 & -0.13 & 0.93 & 0.56 & 0.95 & n.d & n.d \\
\hline 03_Jul_05 & 1365.04 & 0.10 & 0.96 & 0.58 & 0.99 & n.d & n.d \\
\hline 13_Sep_05 & 1364.53 & 0.70 & 0.94 & 0.93 & 0.99 & n.d & n.d \\
\hline 18_Nov_05 & 1364.83 & 0.85 & 0.99 & n.d & n.d & n.d & n.d \\
\hline 03_Apr_06 & 1364.67 & 0.61 & 0.97 & 0.94 & 1.00 & n.d & n.d \\
\hline 14_Aug_06 & 1364.81 & 0.57 & 0.96 & 0.75 & 0.98 & n.d & n.d \\
\hline 14_Nov_06 & 1364.68 & 0.56 & 0.99 & 0.84 & 0.99 & n.d & n.d \\
\hline 12_Dec_06 & 1364.76 & 0.94 & 0.99 & n.d & n.d & n.d & n.d \\
\hline 06_Jan_07 & 1364.97 & 0.86 & 1.00 & n.d & n.d & n.d & n.d \\
\hline 02_Feb_07 & 1364.98 & 0.75 & 0.99 & n.d & n.d & n.d & n.d \\
\hline 01_May_07 & 1364.94 & 0.92 & 0.99 & n.d & n.d & n.d & n.d \\
\hline 18_May_07 & 1365.10 & 0.22 & 0.98 & 0.74 & 1.00 & n.d & n.d \\
\hline 12_Jul_07 & 1365.03 & 0.28 & 0.94 & 0.86 & 0.99 & n.d & n.d \\
\hline 23_Dec_07 & 1365.23 & 0.72 & 0.99 & 0.98 & 1.00 & n.d & n.d \\
\hline 27_Mar_08 & 1365.01 & 0.87 & 0.99 & n.d & n.d & n.d & n.d \\
\hline 28_Mar_10 & 1365.02 & 0.35 & 0.96 & 0.80 & 1.00 & n.d & n.d \\
\hline
\end{tabular}

Table 2. From left to right: Dates of TSI anomalies (January 2003-June 2010; the year is shown with the last two digits), their minimum values, minima and maxima of pressure correlations ( \pm 6 days around TSI minima, approximated to the 2 nd decimal digit; n.d means no data) at Mt. Soro (SO), Stromboli (ST) and Vesuvius (VE). 


\begin{tabular}{|c|c|c|c|c|c|c|c|}
\hline Date & $\begin{array}{c}\text { Forbush } \\
(\%)\end{array}$ & $\begin{array}{c}\mathrm{r}-\mathrm{min} \\
(\mathrm{SO})\end{array}$ & $\begin{array}{c}\mathrm{r}-\mathrm{max} \\
(\mathrm{SO})\end{array}$ & $\begin{array}{c}\mathrm{r}-\mathrm{min} \\
(\mathrm{ST})\end{array}$ & $\begin{array}{c}\text { r-max } \\
(\mathrm{ST})\end{array}$ & $\begin{array}{c}\mathrm{r}-\mathrm{min} \\
(\mathrm{VE})\end{array}$ & $\begin{array}{c}\text { r-max } \\
(\mathrm{VE})\end{array}$ \\
\hline 01_Feb_03 & 45 & 0.91 & 0.99 & 0.86 & 1.00 & n.d & n.d \\
\hline 12_Mar_03 & 25 & 0.41 & 0.98 & 0.84 & 1.00 & n.d & n.d \\
\hline 21_Oct_03 & 55 & 0.56 & 0.99 & 0.62 & 0.95 & 0.62 & 0.97 \\
\hline 28_Oct_03 & 35 & 0.57 & 0.97 & 0.62 & 0.95 & 0.62 & 0.97 \\
\hline 15_Nov_03 & 35 & 0.59 & 0.99 & n.d & n.d & 0.71 & 0.94 \\
\hline 02_Jan_04 & 25 & 0.96 & 1.00 & n.d & n.d & 0.87 & 0.99 \\
\hline 03_Apr_04 & 25 & 0.76 & 0.99 & n.d & n.d & 0.79 & 0.99 \\
\hline 22_Jul_04 & 45 & 0.61 & 0.99 & 0.88 & 1.00 & 0.83 & 0.99 \\
\hline 13_Sep_04 & 55 & 0.75 & 0.97 & 0.87 & 0.99 & 0.74 & 0.95 \\
\hline 05_Dec_04 & 45 & 0.01 & 0.99 & 0.47 & 1.00 & 0.82 & 0.99 \\
\hline 18_Jan_05 & 18 & 0.80 & 0.99 & 0.97 & 1.00 & 0.60 & 0.99 \\
\hline 21_Apr_05 & 17 & 0.48 & 0.95 & n.d & n.d & n.d & n.d \\
\hline 08_May_05 & 55 & 0.52 & 0.97 & n.d & n.d & n.d & n.d \\
\hline 29_May_05 & 45 & -0.45 & 0.88 & n.d & n.d & n.d & n.d \\
\hline 12_Jun_05 & 28 & -0.13 & 0.93 & 0.56 & 0.97 & n.d & n.d \\
\hline 10_Jul_05 & 42 & -0.16 & 0.82 & 0.58 & 0.97 & n.d & n.d \\
\hline 17_Jul_05 & 38 & -0.16 & 0.85 & 0.66 & 0.97 & n.d & n.d \\
\hline 02_Sep_05 & 29 & 0.63 & 0.99 & 0.88 & 1.00 & n.d & n.d \\
\hline 09_Sep_05 & 37 & 0.70 & 0.99 & 0.93 & 0.96 & n.d & n.d \\
\hline 30_Oct_05 & 17 & 0.79 & 0.99 & 0.89 & 0.98 & n.d & n.d \\
\hline 27_Apr_06 & 17 & 0.27 & 0.99 & 0.72 & 0.99 & n.d & n.d \\
\hline 03_Jul_06 & 25 & 0.25 & 0.97 & 0.43 & 0.98 & n.d & n.d \\
\hline 09_Jul_06 & 27 & 0.53 & 0.97 & 0.67 & 0.98 & n.d & n.d \\
\hline 17_Aug_06 & 19 & 0.57 & 0.96 & 0.75 & 0.98 & n.d & n.d \\
\hline 29_Nov_06 & 25 & 0.75 & 1.00 & 0.90 & 1.00 & n.d & n.d \\
\hline 06_Dec_06 & 35 & 0.75 & 0.99 & n.d & n.d & n.d & n.d \\
\hline 14_Dec_06 & 95 & 0.94 & 0.89 & n.d & n.d & n.d & n.d \\
\hline
\end{tabular}

Table 3. From left to right: Dates of Forbush decreases with amplitudes higher than 15\% (January 2003-June 2010; the year is shown with the last two digits), their values, minima and maxima of pressure correlations ( \pm 6 days around the day of Forbush decrease, approximated to the 2nd decimal digit; n.d means no data) at Mt. Soro (SO), Stromboli (ST) and Vesuvius (VE).

amplitudes at Stromboli and Mt. Soro but higher at Vesuvius. During the second period the correlation signal was quite similar at Stromboli and Mt. Soro (no data for Vesuvius), with two correlation minima after 6 days from the secondary and main FDs but with inverted amplitudes (main decorrelation after the secondary FD and vice versa).

The last analytical step consisted in testing our data in the frequency domain, executing a FFT of the 6-day pressure correlation coefficients at Mt. Soro (the only location where data are available without gaps for the two periods). We compared pressure data with the Kp signal, reporting the results in Figure 12. The power spectrum of the pressure correlation signal during the first period, closer to the XIII solar cycle maximum, clearly showed a main peak between 27 and 28 days. This period is the same of the Sun Carrington rotation, equal to 27.2753 days [Wilson 1994] and used for following the periodic reappearance of sunspot groups on Sun's surface. The peak is no more clearly recognizable in the second period. Since the period of the Carrington rotation (27.28 days) is very close to the synodic period of the Moon (27.53 days), the peak observed in the pressure correlation spectrogram could be theoretically 


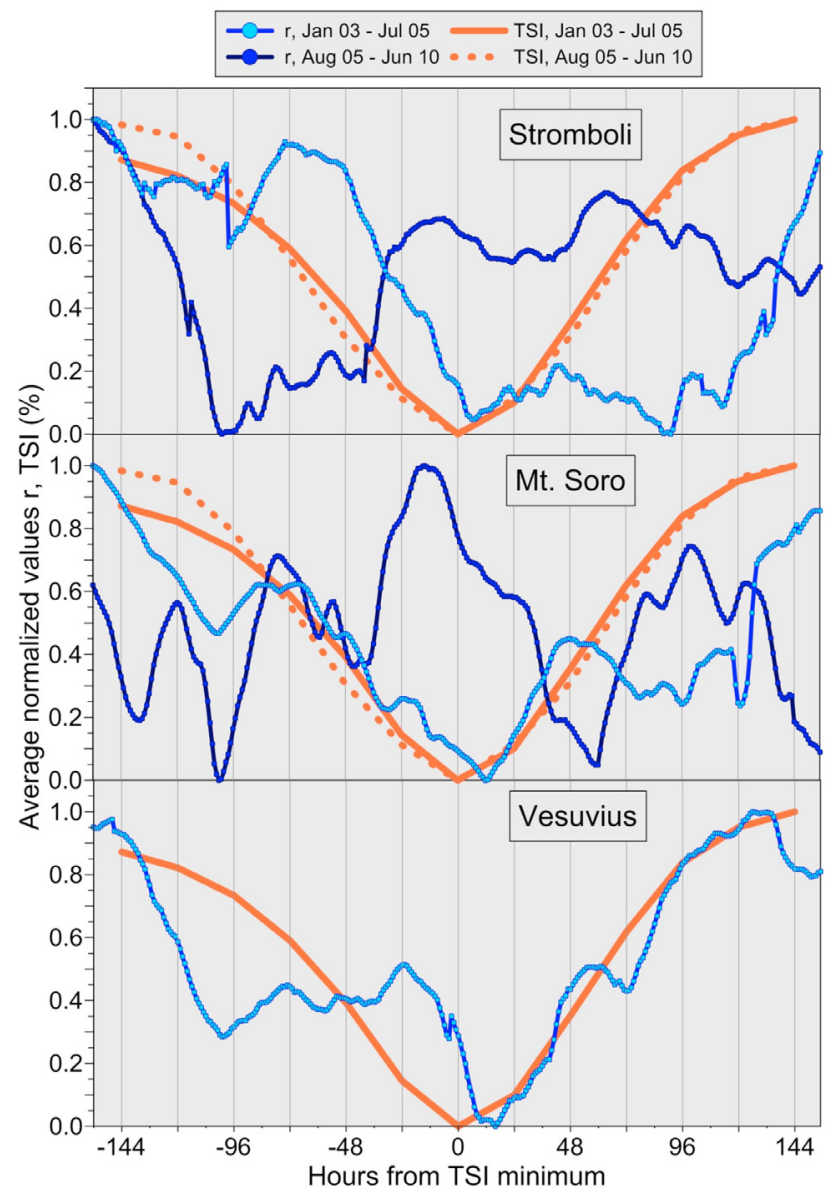

Figure 10. Superposed epoch analysis of pressure correlations (blue lines) around main TSI anomalies (orange continuous and dashed lines). Hour \#0 is $12 \mathrm{AM}$ of the day when the TSI minima were recorded (see Table 2 for the complete list). For clarity of presentation TSI and correlations are presented as normalized values between relative minima and maxima fixed at 0 and 1 respectively.

related to lunar atmospheric tides more than to Sun driven effects. However, two observations contribute to exclude that the peak is related to the Moon phases: i) the effect is observed close to the maximum of the XIII solar cycle (first period) but it is no more clearly visible under a quieter Sun and ii) lunar atmospheric tides have an amplitude of hundredths of hPa [Lindzen and Chapman 1969], two order of magnitude lower than our observed anomalies (several hPa, Figure 4). Moreover, the comparison between $\mathrm{Kp}$ and correlation spectra highlighted several other common features. The most significant of these is the peak at 13.5 days (observed for pressures only during the first period), typical of the geomagnetic disturbances during the declining phases of solar cycles [Love et al. 2012, and references therein].

\subsection{Is space-weather geo-effectiveness responsible for} near ground atmospheric pressure disturbances?

The co-existence of different mechanisms seem to affect the near ground atmospheric pressure regime.

Correlation coefficients between pressures meas-

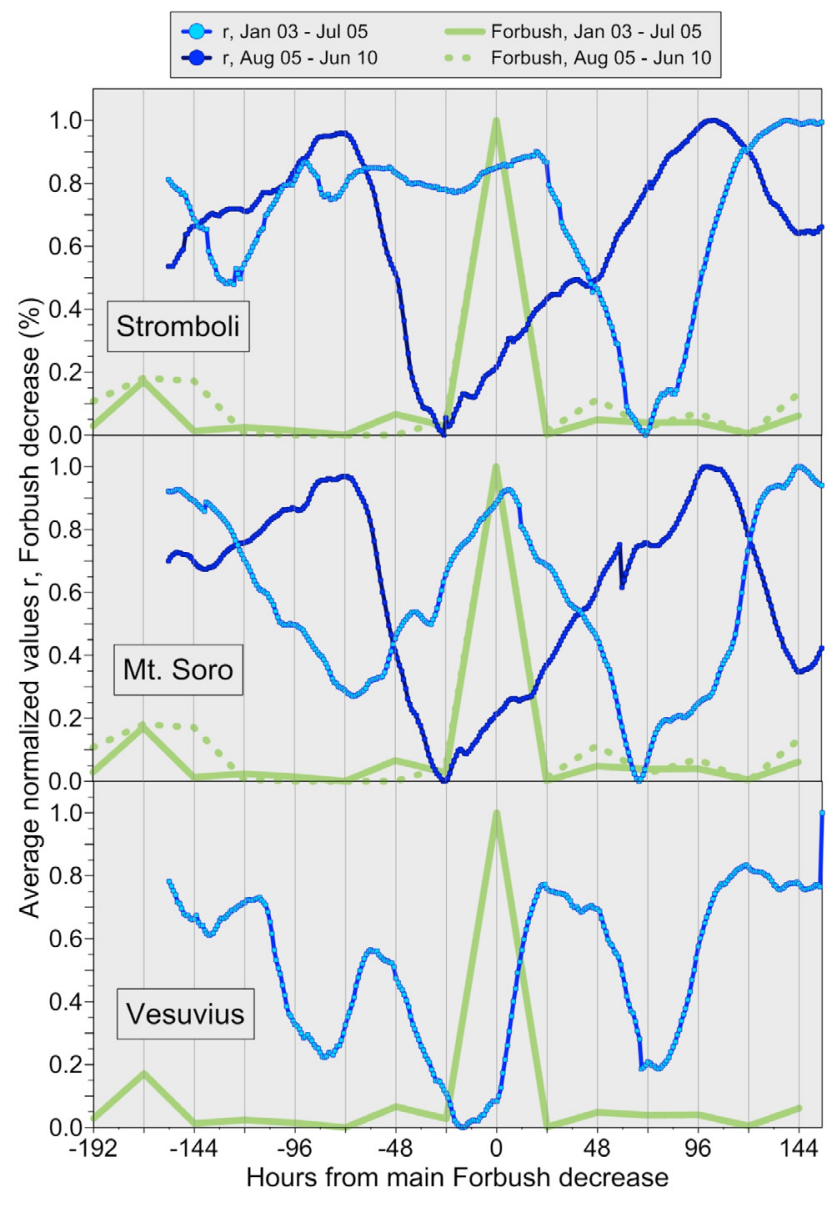

Figure 11. Superposed epoch analysis of pressure correlations (blue lines) around Forbush decreases with amplitudes higher than 15\% (green continuous and dashed lines). Hour \#0 is $12 \mathrm{AM}$ of the day when the main Forbush decreases were recorded (see Table 3 for the complete list). For clarity of presentation FD and correlations are presented as normalized values between relative minima and maxima fixed at 0 and 1 respectively.

ured at different baric heights are modulated by seasonal cycles, with maxima in winter and minima in summer (Figures 3 and 9), evidencing the role of thermal-induced turbulence in near-ground air circulation. As expected, complexity of orographic structures and altitude differences between measuring station play a significant role in controlling correlation drop amplitudes (Figure 3). Simple conic-shaped volcanic islands (Stromboli) show higher average correlation coefficients and lower anomalous drops with respect to volcanic edifice inserted in heavily urbanized areas (Vesuvius) or sectors of complex mountain chains with a huge baric height difference between the stations (Mt. Soro).

An additional role is played by air turbulence induced by volcanic activity, as remarked by the wider pressure differentials (between different baric heights) recorded at Stromboli in 2003 at the end of an effusive eruption (Figure 2). During the same period, more frequent pressure correlation drops were also recorded (Figure 3).

However, astronomic cycles and/or spot volcanic events seem not be the only mechanisms influencing 


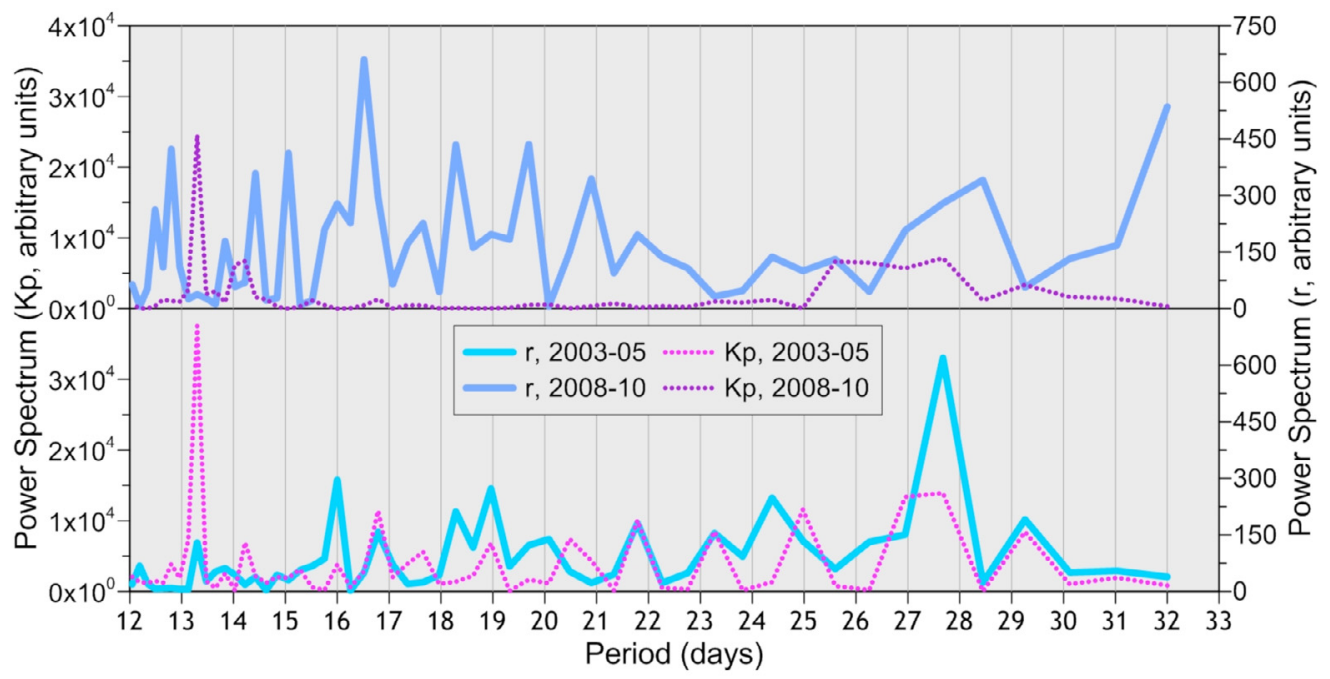

Figure 12. Results of FFT analysis performed on Kp (dotted lines) and 6-days pressure correlation coefficients calculated at Mt. Soro (continuous lines) in the periods January 2003-July 2005 (bottom) and August 2005-June 2010 (top). Since we used a 6-day averaged signal for pressure correlations we started our analysis at 12 days (the minimum value over the Nyquist frequency).

pressure correlations. If the time distribution of correlation drops is compared with the occurrence of changes in Sun radiative flux and/or IMF, leading to relevant geo-effective phenomena, a possible relationship between tropospheric pressure disturbances and Sun's activity is evidenced (Figures 8 and 9). In particular, TSI anomalies seem able to influence the near ground atmospheric pressure field (Figure 10), whereas the role of FDs is not so clear (Figure 11). During periods of high Sun activity TSI and pressure correlations unambiguously followed the same time pattern, with minima of the latter 12 hours after the former. This link is missed during phases of low Sun activity, when pressures changed independently of TSI.

A further support to our convincement is given by the FFT analysis (Figure 12). During periods of high Sun activity, correlation drops showed a main periodicity of about 27 days, the same of the Sun Carrington rotation, and a secondary peak at 13.5 days, typical of the geomagnetic disturbances during the declining phase of a solar cycle. This link is missed again during phases of low Sun activity.

As a final remark, we like to point out that we are not trying to propose a new model for possible tropospheric pressure disturbances induced by Sun activity. The possible models are those proposed in the literature and discussed at the beginning of this chapter. Our work was aimed to present novel data supporting this hypothesis, acquired on ground based acquisition network instead of medium to large scale meteorological observation systems. Near ground pressure correlation drops seem to be a possible proxy for space weather geo-effectiveness, even if this possible relationship can not be presently ascertained out of any reasonable doubt.

\section{Conclusions}

Possible tropospheric pressure disturbances induced by Sun storms are not a novelty. Since the pioneering works after Macdonald and Roberts [1960] and Wilcox et al. [1974], the scientific community is involved in a debate that, lasting for over 50 years, seems not under the way of a near conclusion. The main question is that, although several different observations point to a possible influence of space weather on the Earth atmospheric pressure field, the decisive proof of such a relationship has been not yet found.

The results of our study are lined with the previous observation. Their novelty is related to the measured parameter, i.e. correlation drops between near ground atmospheric pressures seemingly assignable to space weather geo-effectiveness, never investigated before. Their limit, as in the previous studies, the unresolved ambiguity of the final results. Since possible disturbances of the near ground pressure field induced by Sun activity are at least of the same order of those imputed to the "normal" air circulation, it is not easy discriminating between them. A further problem is related to the scarcity of ground-based monitoring networks, specifically designed for acquiring atmospheric pressure data finalised to this field of investigation.

The analysis of new data acquired in correspondence of the present XXIV cycle solar maximum will be a challenging opportunity to strengthen with more independent observations our preliminary results.

Acknowledgements. The research leading to these results has received founding from the European Commissions Seventh Framework Programme under the grant agreement no. 284461 (eHEROES project) and by the ITA MIUR-PRIN grant 2012P2HRCR. It has been also partially founded as a part of the Italian Active Volcanoes Surveillance Program financed by the Italian National De- 
partment for Civil Defence (DPCN). The authors wish to thank Teodoro La Rocca (Meteorological Service of the Italian Air Force) and Pasquale Giugliano (Vesuvius National Park) for the fundamental suggestions and data furnished for the realization of this study. A fundamental help was given by the personnel of the Istituto Nazionale di Geofisica e Vulcanologia (INGV), Sezione di Palermo, involved in the maintenance, under very severe environmental conditions, of the station located at the top of Stromboli volcano: without their hard work our study had never been possible. We are also grateful to two anonymous reviewers, whose suggestions helped us to improve the quality of our work. Last but not least, a very special thank to Sami Solanki, who greatly helped us to correctly develop the pristine idea over which we built up this work.

\section{References}

Brusca, L., S. Inguaggiato, M. Longo, P. Madonia and R. Maugeri (2004). The 2002-2003 eruption of Stromboli (Italy): evaluation of the volcanic activity by means of continuous monitoring of soil temperature, $\mathrm{CO}_{2}$ flux and meteorological parameters, Geochem. Geophy. Geosy., 5 (12), Q12001; doi:10. 1029/2004GC000732.

Kirkland, M.W., and B.A. Tinsley (1996). Are stratospheric aerosols the missing link between tropospheric vorticity and Earth transits of the heliospheric current sheet?, J. Geophys. Res., 101 (D23), 2968929699.

Lindzen, R.S., and S. Chapman (1969). Atmospheric tides, Space Sci. Rev., 10 (1), 3-188.

Love J.J., E.J. Rigler and S.E. Gibson (2012). Geomagnetic detection of the sectorial magnetic field and the historical peculiarity of minimum 23-24, Geophys. Res. Lett., 39, L04102; doi:10.1029/2011GL 050702.

Macdonald, N.J., and W.O. Roberts (1960). Further evidence of a solar corpuscular influence on largescale circulation at $300 \mathrm{mb}$, J. Geophys. Res., 65, 529-534.

Mironova, I., B.A. Tinsley and L. Zhou (2011). The links between atmospheric vorticity, radiation belt electrons, and the solar wind, Adv. Space Res., 50 (6), 783-790; doi:10.1016/j.asr.2011.03.043.

Prikryl, P., V. Rǔsin and M. Rybanský (2009a). The influence of solar wind on extratropical cyclones Part 1: Wilcox effect revisited, Annales Geophysicae, 27, 1-30.

Prikryl, P., D.B. Muldrew and G.J. Sofko (2009b). The influence of solar wind on extratropical cyclones Part 2: A link mediated by auroral atmospheric gravity waves?, Annales Geophysicae, 27, 31-57.

Tinsley, B.A., J.T. Hoeksema and D.N. Baker (1994). Stratospheric volcanic aerosols and changes in airearth current density at solar wind magnetic sector boundaries as conditions for Wilcox tropospheric vorticity effect, J. Geophys. Res., 99 (D8), 1680516813.
Tinsley, B.A., L. Zhou and L. Weiping (2012). The role of volcanic aerosols and relativistic electrons in modulating winter storm vorticity, Adv. Space Res., 50 (6), 819-827; doi:10.1016/j.asr.2011.12.019.

Wilcox, J.M., P.H. Scherrer, L. Svalgaard, W.O. Roberts, R.H. Olson and R.L. Jenne (1974). Influence of solar magnetic sector structure on terrestrial atmospheric vorticity, J. Atmos. Sci., 31, 581-588.

Wilson, P.R. (1994). Solar and stellar activity cycles, Cambridge Astrophysics Series, Cambridge, MA: Cambridge University Press.

\footnotetext{
${ }^{\star}$ Corresponding author: Paolo Madonia, Istituto Nazionale di Geofisica e Vulcanologia, Sezione di Palermo, Palermo, Italy; email: paolo.madonia@ingv.it.

(C) 2014 by the Istituto Nazionale di Geofisica e Vulcanologia. All rights reserved.
} 